\title{
Article
}

\section{Interventions to reduce unnecessary caesarean sections in healthy women and babies}

Betrán, Ana Pilar, Temmerman, Marleen, Kingdon, Carol, Mohiddin, Abdu, Opiyo, Newton, Torloni, Maria Regina, Zhang, Jun, Musana, Othiniel, Wanyonyi, Sikolia Z, Gülmezoglu, Ahmet Metin and Downe, Soo

Available at http://clok.uclan.ac.uk/24459/

Betrán, Ana Pilar, Temmerman, Marleen, Kingdon, Carol ORCID: 0000-00025958-9257, Mohiddin, Abdu, Opiyo, Newton, Torloni, Maria Regina, Zhang, Jun, Musana, Othiniel, Wanyonyi, Sikolia $Z$ et al (2018) Interventions to reduce unnecessary caesarean sections in healthy women and babies. The Lancet, 392 (10155). pp. 1358-1368. ISSN 0140-6736

It is advisable to refer to the publisher's version if you intend to cite from the work. http://dx.doi.org/10.1016/S0140-6736(18)31927-5

For more information about UCLan's research in this area go to http://www.uclan.ac.uk/researchgroups/ and search for <name of research Group >.

For information about Research generally at UCLan please go to http://www.uclan.ac.uk/research/

All outputs in CLoK are protected by Intellectual Property Rights law, including Copyright law. Copyright, IPR and Moral Rights for the works on this site are retained by the individual authors and/or other copyright owners. Terms and conditions for use of this material are defined in the policies page.

\section{CLoK}

Central Lancashire online Knowledge www.clok.uclan.ac.uk

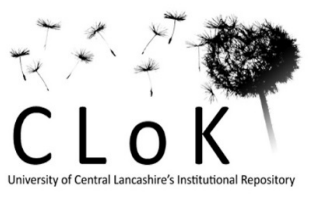


1 Optimising Caesarean Section Use 3. Interventions to reduce unnecessary

2 caesarean sections in healthy women and babies

3 Ana Pilar Betrán, Marleen Temmerman, Carol Kingdon, Abdu Mohiddin, Newton Opiyo, Maria

4 Regina Torloni, Jun Zhang, Musana Othiniel, Sikolia Z Wanyonyi, A Metin Gülmezoglu, Soo Downe

5

6 UNDP/UNFPA/UNICEF/WHO/World Bank Special Programme of Research, Development and

7 Research Training in Human Reproduction, Department of Reproductive Health and Research,

8 World Health Organization, Geneva, Switzerland

9 (AP Betrán PhD, AM Gülmezoglu PhD);

10 Aga Khan University, Department of Obstetrics and Gynaecology, Nairobi, Kenya, and Ghent

11 University, Faculty of Medicine and Health Sciences, Ghent, Belgium

12 (M Temmerman PhD, professor, A Mohiddin MbChB);

13 School of Community Health and Midwifery, Faculty of Health and Wellbeing, University of Central

14 Lancashire, Preston, United Kingdom

15 (C Kingdon PhD, S Downe PhD, professor);

16 Review Production and Quality Unit, Cochrane, London, United Kingdom

17 (N Opiyo PhD);

18 Evidence Based Healthcare Post Graduate Program, São Paulo Federal University, São Paulo, Brazil 19 (MR Torloni PhD);

20 MOE-Shanghai Key Laboratory of Children's Environmental Health, Xinhua Hospital, Shanghai Jiao

21 Tong University School of Medicine, Shanghai, China

22 (J Zhang PhD);

23 St Francis hospital Nsambya/Uganda Martyrs University, Kampala, Uganda

24 (M Othiniel MbChB);

25 Aga Khan University, Department of Obstetrics and Gynaecology, Nairobi, Kenya

26 (SZ Wanyonyi MD); 
29

30

- Optimising caesarean section (CS) is of global concern. Underuse leads to higher perinatal mortality and morbidity and should remain a global health priority.

- Conversely, high rates of CS have not shown benefits, can be harmful and can commit resource use unnecessarily. Addressing overuse therefore also needs to be the focus of reaching optimum levels of CS around the world.

- Few clinical interventions have been tested in randomised trials with CS as a primary outcome. Although labour induction at or near term may reduce CS, the side effects, costs, and service user and provider acceptability of routine labour induction with no medical indication have not been established. Trials that include continuous labour support show similar reductions in CS rates to those of labour induction.

- Trials of non-clinical healthcare interventions suggest that approaches that prioritise positive human relationships, promote respectful and collaborative multidisciplinary teamwork and address clinician beliefs and attitudes, and women's fear of labour pain and of poor quality of care, might be effective in reducing CS or increasing physiological labour and birth. These include labour companionship, midwife-led continuity of care, midwife-led units, antenatal education, training and implementation of evidence based guidelines at the point of care along with mandatory second opinion and timely feedback to staff.

- Multifaceted (clinical and non-clinical) strategies are needed to reduce CS and/or to increase physiological birth for healthy women and babies. These must be scientifically tested, and tailored to local determinants (beliefs, norms, and behavioural factors that influence the key players, i.e. societal norms, women, health professionals, and healthcare organisations).

- Further research is needed to evaluate the effect of CS overuse on resolving concomitant underuse.

\section{Summary}

Optimising caesarean section (CS) is of global concern. Underuse leads to higher maternal and perinatal mortality and morbidity. Conversely, CS high rates have not shown benefits and can create harm. Worldwide, CS rates continue to escalate and interventions to reduce unnecessary CS have shown limited success. Identifying the underlying factors for the continued rise could improve the efficacy of interventions. We describe the factors associated with women, families and societies; health professionals; and organisations and systems. We examine behavioural, psychosocial, health systems and financial factors. We outline the type and effect of interventions that have been subject to research to reduce CS rates. Clinical interventions such as external cephalic version for term breech, vaginal breech delivery in well selected cases, and vaginal birth after CS (VBAC), may 
contribute to reduce CS rates. Approaches such as labour companionship and midwife-led care have been associated with higher rates of physiological birth, safe outcomes, lower healthcare costs, and

68 positive maternal experiences in high-income countries. Such approaches need assessment in

69 middle- and low-income countries. Educational interventions for women must be complemented with meaningful dialogue with health professionals, and effective emotional support. Investing in

71 health professionals, eliminating financial incentives, and reducing fear of litigation is fundamental.

72 Safe, private, welcoming, adequately resourced facilities are needed. At country level, effective leadership is essential to ensuring CS use only when needed. We conclude that interventions to reduce overuse must be multi-component and locally tailored, addressing women's and health professionals' concerns, and health system as well as financial factors.

This paper is the third in a three-part Series on optimising CS rates, and focuses on interventions to reduce unnecessary $\mathrm{CS}$. 
There is no debate about the need to increase access to safe CS where the procedure is underused. However, there is no evidence of benefit for women and babies who do not require the procedure, ${ }^{1}$ and, as for any surgery, there are short- and long-term risks that have been outlined in the second paper of this Series. ${ }^{2}$ In addition, where surgery is overused, this may limit resources that could be used to increase underuse. ${ }^{3}$

The optimal population-level CS rate escapes consensus. ${ }^{4}$ Even the intent to develop a global standard is contested. However, there is almost universal consensus that, in many settings, current rates cannot be medically justified..$^{5-8}$ Underuse of CS has been the focus of literature, research, policy and funding efforts over decades, since lack of access to CS is a priority to reduce maternal and perinatal mortality and morbidity. Overuse, on the other hand, is a more recent and less well understood phenomenon that can coexist in many countries with underuse. ${ }^{9}$ There may be the potential, therefore, to free up resources to address such underuse. This third paper in this Series on optimising CS rates is thus focused on interventions to reduce unnecessary CS, which we define as being performed in the absence of medical (including psychological) indications. ${ }^{10-12}$

We begin with an overview of the drivers behind increasing CS rates. We then examine the nature and effects of both clinical and non-clinical (behavioural, educational, psychosocial) interventions that have been tested in studies specifically designed to safely reduce CS births. We discuss the degree to which these interventions pay attention to the underlying drivers, and the mechanisms of effect that might underpin successful reduction strategies. Finally, we propose research priorities for the future.

Drivers of "too many caesarean sections"

Many decisions to undertake CS are driven by the clinical or psychological needs of the mother and/or baby. However, where rates are higher than needed, the drivers fall into three broader categories that sometimes overlap, and are interconnected. These relate to: 1 ) childbearing women, families, communities, and the broader society; 2 ) health professionals; and 3) healthcare systems, financing, and organisational design and cultures.

Factors related to childbearing women, families, communities, and the broader society

111 The notion of maternal request for CS has been variously interpreted, and widely debated. ${ }^{13-15}$

112 Contrary to perceived opinion, however, the majority of women around the world do not prefer a CS 113 in the absence of current or previous complications. ${ }^{16,17}$ The most recent systematic review on 114 worldwide preferences reported an overall pooled preference for CS of $15 \%$, and only $10 \%$ when excluding women with a previous $\mathrm{CS}^{16}$

116 For women who do favour CS in the absence of medical indications, reasons include fear of labour 117 pain, particularly where epidural analgesia is not accessible or affordable, ${ }^{18-24}$ fear of pelvic floor 
damage and urinary incontinence, ${ }^{25,26}$ or fear of a reduced quality of sexual life. ${ }^{26-28}$ Contrary to scientific evidence, most women who prefer CS perceive it to be safer for the baby and for themselves. ${ }^{29-32}$ Less commonly, women cite convenience as a reason, particularly in societies where women bear substantial work or family responsibilities, ${ }^{24,29}$ or where they can also have a tubal ligation. ${ }^{19,26,33}$ In some settings, there are perceived advantages for the child of an auspicious birthdate. ${ }^{18,24,28,34}$ Previous negative experiences of vaginal birth, including suboptimal quality of care, and experiences of disrespect and abuse, also influence choice for CS birth in subsequent pregnancies. ${ }^{23,35-37}$

Contemporary society exposes pregnant women to a wide range of information on pregnancy and childbirth. ${ }^{38-41}$ The media has a growing influence on the decision for elective CS. ${ }^{15,41-43}$ Elective CS birth tends to be presented as controllable, convenient, more fashionable and modern. ${ }^{40-42} \mathrm{~A}$ few studies suggest that the influence of fathers' preference is related to convenience, previous negative experience of a partner's labour or birth, or previous experience of a partner having a CS. ${ }^{44}$

\section{Factors related to health professionals}

Pregnant women tend to identify health providers as the most important influence on their decision about mode of birth. ${ }^{24,45,46}$ In contrast, health providers report women's request as an important driver for performing non-medically indicated CS deliveries. A pan-European survey of 1530 obstetricians found compliance with a hypothetical women's request for CS without medical indications to be lowest in Spain (15\%) and highest in the UK (79\%). Higher levels of fear of litigation, employment in a university-affiliated hospital, and being male, were all associated with an increased likelihood of agreement to a woman's CS request. ${ }^{6}$

In many countries, malpractice legal standards and systems leave providers vulnerable even if they deliver the best evidence-based care. ${ }^{47}$ Contrary to scientific evidence, society in general believes that a CS is a protective procedure. ${ }^{48,49}$ Consequently, practitioners are more likely to be sued for complications during vaginal delivery than for unnecessary $\mathrm{CS}$, even if there is no evidence of error. ${ }^{47,48}$ Being sued (even if unsuccessfully) can generate negative publicity, damage reputations and professional confidence, and even destroy careers. ${ }^{50-53}$ This situation may result in performing a CS for professional protection, rather than to benefit the mother and/or baby. ${ }^{54}$

In some settings, most CS operations occur during working hours, and during weekdays, peaking on Fridays. ${ }^{55,56}$ This suggests that the decision to perform a CS sometimes occurs for convenience. In settings where obstetricians combine public and private work, scheduling elective CSs allows for private work to be reconciled with public duties. ${ }^{34,53,57}$

\section{Factors related to healthcare systems, financial reimbursements, and organisational design and cultures}

In many but not all settings, the rates of CS birth are higher in the private sector. ${ }^{58}$ In Brazil, for example, $80-90 \%$ of all babies in the private sector are born by CS, compared with $30-40 \%$ in the public sector. ${ }^{59,60}$ In some settings, private maternity care underpins the finances for whole hospitals. Where a CS can generate more income than a vaginal birth, incentives exist to persuade women that a CS is best for her and/or her baby. ${ }^{57}$ 
Lack of experience or skills in performing an assisted vaginal delivery has been associated with higher CS rates, ${ }^{53}$ especially in settings where there is lack of training and supervision, and young physicians are afraid of showing signs of incompetency, or of 'disturbing' senior staff to ask for support. ${ }^{53}$ In many settings, young obstetricians have become experts in CS, but are losing confidence in undertaking vaginal assisted deliveries and breech deliveries.

Women's experiences of poor quality antenatal environments, equipment, and health professional skills and interactions are associated with a lack of trust in the system and staff. This can trigger a decision to undergo a CS to avoid anticipated poor-quality labour and birth care. ${ }^{24,37}$ In some lowresource settings, high CS rates in tertiary hospitals have been attributed to unskilled primary care professionals, who delay referral because they fail to detect danger signs. The transferred woman arrives late and in a critical condition and an emergency CS is the only solution. ${ }^{53}$

Figure 1 shows a schematic representation of all the non-clinical factors discussed above enclosing the obstetric and clinical factors that affect the rate of CS births (e.g. presentation, number of foetuses, previous (S), represented in the middle by the Robson's 10 -group classification. ${ }^{61,62}$ It is intended to visualise the layers of complexity of the factors involved.

\section{Interventions to reduce unnecessary caesareans}

Interventions to reduce unnecessary CS can be broadly conceptualised as clinical and non-clinical, though there is overlap between the two. The former tend to target a specific clinical practice for a particular woman (e.g. vaginal birth after CS, VBAC). The degree to which such interventions can reduce the CS rate may be limited because CS for clinical indications forms an increasingly smaller proportion of the overall rising rate that has been confirmed by the findings of the first paper in this Series. ${ }^{8}$ The latter tend to address one or more aspects of the system of care design and/or delivery, and to be more multifactorial. The World Health Organization (WHO) has recently produced guidelines on antenatal and intrapartum care, ${ }^{63,64}$ and these include recommendations on some of the clinical interventions that reduce CS and improve other outcomes for mother and baby. These and other clinical interventions are summarised below, followed by a more in-depth analysis of nonclinical interventions. ${ }^{65}$

\section{Clinical interventions}

Only two clinical interventions for healthy women and babies with no complications have been tested in randomised trials with a primary outcome of intrapartum (emergency) CS: routine induction of labour at or near term, and active management of labour. The routine induction trials either found no difference in CS rates ${ }^{66}$ or reduced rates of CS. ${ }^{67-69}$ The latest Cochrane review on this topic did not have mode of birth as a primary outcome, but did show a reduction in CS overall, ${ }^{70}$ as did the recently completed ARRIVE trial of over 6000 low risk primigravid women randomised at or around 39 weeks' gestation (RR $0.84,95 \% \mathrm{Cl} 0.76$ to 0.93 ). ${ }^{71}$ However, some induction-of-labour studies report increased rates of instrumental birth, ${ }^{67-70}$ and women's views and experiences are rarely reported. In addition, recruitment rates seem to be low; for example, about a third of eligible women agreed to take part in the 35/39 trial, a randomised, controlled trial of primigravid women 
35 years of age or older assigning women to labour induction at 39 weeks or to expectant management, ${ }^{66}$ and $25 \%$ of those eligible agreed to take part in the ARRIVE trial. ${ }^{72}$ This raises questions about women's willingness to undergo labour induction, and, consequently, about the external generalisability of the findings.

The most up to date Cochrane review of active management of labour (strict diagnosis of labour, routine amniotomy, oxytocin for slow progress, and one-to-one support in labour) shows no statistically significant difference in CS rates. ${ }^{73}$ However, when authors excluded one low-quality study, the results showed a statistically significant reduction ( $R R 0.77,95 \% \mathrm{Cl} 0.63$ to 0.94 ). It has been suggested that the primary mechanism could be the one-to-one support component. ${ }^{73}$ This hypothesis is strengthened by the most recent Cochrane review on continuous labour support, which found that continuous labour support may reduce CS rates ( $R R=.75,95 \% \mathrm{Cl} 0.64,0.88$ ), although CS was measured as secondary outcome. ${ }^{74}$

Although external cephalic version (ECV) is used to reduce CS in breech presentation, it does not appear to do so in trial conditions. ${ }^{75,76} \mathrm{CS}$ rates for breech presentation rose to near $100 \%$ in many settings following the publication of the results of the Term Breech Trial, ${ }^{75,77}$ but the authors of the two-year trial follow-up noted that 'planned cesarean delivery is not associated with a reduction in risk of death or neurodevelopmental delay in children at 2 years of age'. ${ }^{78}$ Some centres are now offering carefully screened women the option of trying for a vaginal breech birth, with generally good outcomes. ${ }^{79}$

Women with a previous CS but who have no complications in a subsequent pregnancy, are often offered a trial of labour with a view to achieving VBAC. The only trial of VBAC in the relevant Cochrane review includes data on mode of birth for just 22 women, with no significant difference in CS rates ${ }^{80} \mathrm{~A}$ recent large European trial of this approach is due to report soon. ${ }^{81}$ The influence of medical opinion leaders might improve the uptake of VBAC and consequently reduce CS rates. ${ }^{82}$ Despite the lack of trial evidence on mode of birth, both ECV and VBAC are part of usual clinical practice in many settings, since they are associated with other benefits for mother and baby..$^{83,84}$

Apart from studies with a primary outcome of reducing CS rates, randomised trial evidence suggests that limiting the "cascade" of interventions that women and babies are sometimes exposed to can increase rates of spontaneous vaginal birth. This may be achieved by midwifery led continuity of care; ${ }^{85}$ planning labour in birth centres (in settings where there is access to rapid transfer); using intermittent auscultation rather than electronic fetal monitoring; ${ }^{86}$ and continuous labour support. ${ }^{74}$

The evidence considered in the context of the drivers discussed above suggests that meaningful reductions in CS rates cannot be achieved by clinical interventions alone. Non-clinical interventions are more likely to be synchronous with these behavioural and psychosocial drivers.

\section{Non-clinical interventions}

Acknowledging that there is no clear dividing line between clinical and non-clinical interventions, we defined non-clinical interventions as those that are applied independently of a clinical encounter between a specific healthcare provider and a particular service user. ${ }^{65}$ Recent WHO recommendations on this topic (web appendix Table 1) drew on an updated Cochrane review (29 included studies), ${ }^{12}$ and three qualitative evidence syntheses on women's and providers' views, 
values, beliefs and perceptions about CS, and factors related to organisations, facilities and systems (49 studies reported in 52 papers). ${ }^{45,87,88}$ Below we present a summary of the findings of these reviews.

\section{Interventions targeted at women, families and communities}

241 Among other drivers identified above, fear, concerns about safety, convenience, and mis-

242 information, and wider society/peer group norms were all relevant for decision making about mode of birth for women and families. The effectiveness evidence in this area is derived from 12 randomised controlled trials, all comparing specific education, support programmes, and birth preparation classes with usual practices, and mostly addressing knowledge, anxiety and fear. ${ }^{12}$ Three interventions (web appendix Table 2) (tested in small studies with fewer than 200 participants each) reduced CS births: nurse-led applied relaxation training programme (Iran) ${ }^{89}$ psychosocial couplebased prevention programme (United States), ${ }^{90}$ and childbirth training workshop (Iran). ${ }^{91}$ In addition, one Finnish study of 371 women with fear of childbirth reported no significant effect on the overall rate of CS but a $33 \%$ increase in spontaneous vaginal births. ${ }^{92}$ All were low-quality, single-site studies.

Three studies assessed different formats of educational intervention in women with a previous CS: role play education versus lectures in nulliparous women (Iran); ${ }^{93}$ interactive decision aids versus educational brochures (USA), ${ }^{94}$ and individualised prenatal education and support versus written information pamphlets (Canada). ${ }^{95}$ None of these three studies showed significant differences in rates of CS or VBAC. ${ }^{12}$

The qualitative evidence synthesis was based on 12 studies published between 2001 and 2016, undertaken in Australia, Brazil, Canada, Norway, Taiwan, UK and USA, in mostly urban settings. ${ }^{45}$ The studies encompassed both highly motivated women who expressed an intense desire for engagement, and those who wanted the provider to make relevant decisions. Across all groups, pregnant women welcomed educational interventions. They reported that new knowledge could be empowering, informing more meaningful dialogue with providers, assuming the content and format did not provoke anxiety. Women welcomed online and digital information, but many still wanted printed copies to reflect on, and to revisit with family and friends. ${ }^{96}$

Face-to-face dialogue with health professionals was reported to be a strong influence on decisions about birth mode, especially when clinicians recognised childbirth as an emotional experience, rather than just a clinical process. Frustration and mistrust resulted when women felt they were not listened to, or that advice provided was inconsistent.

\section{Interventions targeted at health professionals}

270 Concerns about litigation, organisational and peer group norms, and financial benefits and convenience were identified above as drivers of health professional use of CS in some cases. Interventions directed at health professionals have included educational packages to improve adherence to evidence-based clinical practice, second-opinion policies, audit and feedback, and peer-review of CS indications. ${ }^{12}$ Two interventions were found to slightly reduce the CS rate with high-certainty evidence (web appendix Table 2): implementation of evidence-based guidelines combined with structured, mandatory second opinion (sites in Argentina, Brazil, Cuba, Guatemala 
and Mexico), ${ }^{97}$ and implementation of evidence-based guidelines combined with CS audits and timely feedback (multiple sites in Canada). ${ }^{98}$

Qualitative evidence synthesis of health professionals' views and experiences of non-clinical interventions to reduce unnecessary CS included 17 studies (2005-2017) from 17 countries (Australia, Canada, China, Ethiopia, Finland, Germany, Iran, Ireland, Italy, Kenya, Netherlands, Nicaragua, Sweden, Tanzania, Uganda, UK and USA), in both rural and urban settings. ${ }^{88}$

Health professionals' beliefs about birth (on a continuum from considering it a normal physiological process to inherently pathological) informed both their knowledge about what constitutes necessary and unnecessary CS, and the importance they attached to reducing overuse. Some obstetric residents reported a need for improved communication, while also fearing that seeking a second opinion could negatively impact on their clinical credibility and career. Some professionals were opposed to second-opinion policies because of consequent difficulties in medico-legal responsibilities. A few welcomed guidelines as providing a defendable basis for their practice (rather than as a basis for good practice per se), while others resisted guideline-directed practice, believing that they only intervened when necessary.

Responses to interventions like audit and feedback were influenced by fear of blame and recrimination, the value attached to personal financial reward, preference for CS as an efficient birth method that can be scheduled, and beliefs about women (including their perceptions of women's preparedness to give birth vaginally, lack of antenatal education, sedentary lifestyles and increasing rates of obesity). Doubts about local validity of guidelines, keeping them up-to-date and lack of resources limited implementation of guidelines. In European settings, health professionals experienced interventions targeted at overuse as most acceptable where this vision was shared within and between multidisciplinary groups, and when they felt supported by colleagues and opinion leaders.

\section{Interventions targeted at organisations, facilities, and systems}

As noted above, the drivers for CS at the systems level included financing and care-provision models, system integration, and environmental and resourcing conditions. Interventions at this level to reduce CS births include changes in organisational culture, insurance reforms, external peer review, legislative policy limiting legal liability in case of litigation, facility staffing models, specific goals for CS rates, and targeted financial strategies. ${ }^{12}$

Three studies were identified (web appendix Table 2) ${ }^{65} \mathrm{~A}$ single-site study in the USA tested a change in the model of care ${ }^{99}$ by switching privately insured women from a physicians' privatepractice approach to a model of care provided primarily by midwives with 24-hour in-house obstetrician back-up without other competing clinical duties. This led to a significant decrease in the rate of primary CS and an increase in VBACs. The other two studies were of very low quality (uncertain evidence). They assessed financial incentives for health professionals. In one hospital in the USA, equalising physician fees for vaginal and CS delivery resulted in a non-significant reduction in CS rates. ${ }^{100}$ In Taiwan, the National Health Insurance scheme raised the fee for a vaginal birth to the level of CS, without a significant effect on CS rates. ${ }^{101}$ 
316 Qualitative evidence synthesis in this area included 25 studies (1993-2016) from 17 countries (nine

317 from Europe or North America, five from Africa, four from Latin America, three from China, two from

318 Iran, one from Bangladesh, and one from Lebanon), in rural and urban settings. ${ }^{87}$ Some participants

319 worked in settings where the organisational culture endorsed maternal request for CS, and/or where

320 there was the belief that quality of care was compromised by reductions in CS rates. In other

321 settings, reducing CS rates was believed to enhance overall quality of care. This influenced whether

322 changes in the physical birth ambience to encourage labour and vaginal birth were properly

323 maintained or not, and whether any change was followed or ignored by staff.

324 There was a consistent message across studies and countries that the birth environment was an

325 amalgam of both the physical structures and resources, and of the state of relationships between

326 professionals and stakeholders, all contributing to a sense of the organisational ethos. In Iran,

327 Lebanon and Nicaragua, substandard conditions in maternity care were reported as significant

328 barriers to reducing unnecessary CS. Critically, the balance of power between doctors, midwives,

329 nurses, other maternity care providers, and childbearing women strongly influenced willingness to

330 engage or not with improving the organisational ethos. Respectful multidisciplinary teamwork and

331 communication seemed to be fundamental to promoting efforts to reduce CS rates. ${ }^{87}$

333 Unpacking mechanisms of effect

\section{What makes the difference?}

335 The data we present suggest that very few interventions (clinical or non-clinical) have paid attention

336 to the multiple drivers of high CS rates and their interactions ${ }^{45,87,88}$ which are complex, dynamic, and,

337 to an extent, context-specific. As a consequence, very few have been effective in reducing

338 unnecessarily high CS rates. ${ }^{7,12}$ For example, addressing preparedness and knowledge of pregnant

339 women while ignoring healthcare providers' demand for skills and training or for more pro-vaginal

340 policies on birth malpractice is unlikely to reduce CS births. In addition, the interactions between

341 factors may require continuous adaptations and change. For example, positive reputation and

342 respectful relationships between different cadres or between providers and pregnant women and

343 communities need time, effective communication and understanding. Incremental adaptations may

344 result in more sustainable results than drastic artificially imposed changes in already tense

345 environments.

346 Interventions for women need to engender a sense of empowerment. They need to be implemented

347 in conjunction with meaningful dialogue with health professionals and with those who set maternity

348 care norms in local communities. They should include recognition of previous experience of birth,

349 short- and long-term effects of CS on women and children and be provided in the context of

350 effective emotional support.

351 Professional norms, beliefs and values that influence local decision making in practice tend to

352 operate independently of (and sometimes despite) the known evidence base. Mutually respectful

353 multidisciplinary teamwork where all staff groups are authentically working to optimise positive,

354 safe childbirth seems to be a characteristic of some services that have safely reduced CS rates, as

355 part of an overall programme of good-quality care. Barriers to effective collaboration and 
communication need to be removed to increase the chances of success. Appropriate management of the change process, including changes in work patterns, workloads, skills, and professional ethos that some new approaches require, is also crucial.

The importance of effective, tailored and continuous professional education, training, and support cannot be overstated. Staff need to have, and maintain, the skills to provide flexible support for individual women in their pursuit of safe normal or vaginal instrumental birth, and good decision making about when interventions are needed along with continuous quality improvement. This is essential to reduce fear of litigation.

Health systems are the hardware supporting or undermining clinical and professional efforts for change. The overall organisational ethos is important, and needs to be understood and addressed. The creation of safe, private, welcoming, adequately resourced labour and birth environments that optimise a sense of being relaxed and supported for women and for health professionals is essential.

Notably, the first step for success is local recognition of the problem. Changes externally imposed on facilities and professionals are a recipe for failure. Participatory approaches are more likely to be effective. At both country and facility level, strong and responsive leadership and authentic, sustainable commitment to reduce unnecessary CS are crucial. The reduction of overuse requires a change in organisational mentality and needs high visibility. Bringing this to the attention of the public requires production and distribution of printed, audio-visual, and virtual material as well as social and mass media coverage.

\section{Implementing change effectively}

While all the factors discussed in this article are essential elements in the equation, their sheer volume can seem overwhelming (Figure 1). Implementation and improvement science offers a range of theories to operationalise effective interventions. ${ }^{102-104}$ Successful initiatives for complex health systems have used participatory methods and action-led processes to build audit and change cycles into the intervention process. ${ }^{105,106}$ These implementation mechanisms allow for more flexible designs to integrate local barriers and mediators while still incorporating population-level evidence. They overcome the sense of helplessness that can arise when faced with the apparent complexity of insufficient human resources or materials, suboptimal communication, toxic power relations, perverse financial incentives, and adverse professional and societal norms. When compared with simple, linear, top-down interventions that demand fidelity to very specific components, participatory approaches and subsequently adapted actions identify exactly where in the local system change is possible, and adopt multiple interventions that address all the locally relevant blocking factors. ${ }^{104-106}$ Indeed, recent evidence from specific sites and regions in China suggests that using multi-level interventions that include change in government policy, financial incentives, local benchmarking, education of staff and of service users, provision of doula support and access to pain relief, can limit the rise in CS rates. ${ }^{107,108}$

\section{Future research priorities}

With some exceptions, interventions tested to date to reduce unnecessary CS have been singlefaceted, targeted to one group (e.g. women or healthcare providers), tested in a single site or country with a relatively small number of participants, and providing low- or very low-quality 
evidence. Women's views and experiences were often not included, and medium- and long-term follow up was not undertaken. Studies have rarely considered the qualitative evidence of what might work for a particular barrier or facilitator. Future interventions are unlikely to be effective if they repeat these errors. The consideration of the local context, culture, norms, practice and pre-existing initiatives is essential not only for the optimal design of the intervention and its components but also as mediators to negotiate and overcome resistance to change. ${ }^{109-111}$

Supportive models of care, including labour companionship, ${ }^{74}$ midwife-led continuity of care,${ }^{85}$ and midwife-led units, ${ }^{112}$ provide promising approaches. They tend to prioritise positive human relationships, and to optimise physiological labour and birth for healthy women and babies. In trials in high-income countries, these models have also been associated with safe outcomes, lower healthcare costs, and positive maternal experiences for both healthy women and babies, and those with complications. The feasibility and applicability of such approaches now need to be assessed in middle- and low-income countries.

Research is also needed to evaluate the effect of overuse of CS on resolving concomitant underuse. Promising ideas that could optimise both over- and underuse, but that require more research, include ways of changing organisational ethos and culture, of maximising respectful intra- and interprofessional teamworking, and provider-women relationships; financial interventions that may involve physicians individually but also hospitals or whole systems; maintaining reimbursement costs of vaginal delivery close to those for CS or even higher; establishing targets for CS rates at facility level; or public dissemination of CS rates by hospital. ${ }^{109,110,113-115}$

Across ideas, complex interventions are challenging to develop, evaluate, document and reproduce, and are subject to more variation than a drug. ${ }^{109,116}$ Many complex interventions are implemented in contexts that prioritise action over generation of evidence. ${ }^{104,110,113,117}$ In general, future research in this area should be based on well-designed participatory and action-focused studies that unify the rigour of research and the flexibility needed to optimise complex multifaceted interventions. ${ }^{104,105}$ Proper evaluation of the effectiveness of any intervention before widescale implementation takes place is critical. Additionally, new interventions need to be designed and tested, based on the drivers for higher or lower rates of CS identified in the qualitative data presented in this paper.

Among the few studies that exist in this area, even fewer are based in low-income countries, though these are also experiencing rising rates of unnecessary CS births in parallel with underuse. ${ }^{9}$ Future studies should also address drivers and interventions relevant to these other countries, where inequities in the use of CS are more prevalent, and detrimental effects much higher. ${ }^{9}$

\section{Conclusion}

Although there is almost universal consensus that current CS rates have transgressed reasonable justification of need, effective interventions to optimise CS births by increasing underuse and reducing overuse have proven elusive. Their limited success may be due to the complexity of the factors driving the under- and the overuse of CS worldwide and the prevalent approach in research to focus on single interventions that target only one driver. Given the issues discussed in this paper, 


\section{Authors' contributions} systems. and timely feedback to staff.

\section{Competing interests}

None

\section{Disclosures}

None

addressing overuse is critical to optimising maternity care experiences and outcomes. New multicomponent interventions that can be tailored to local contexts and drivers should be devised to address the concerns of women and health professionals, as well as the limitations of health

A CS is not a stand-alone event, and it is not intrinsically an adverse outcome. Indeed, reducing CS below safe levels, or replacing it with badly performed instrumental birth, is more likely to cause harm than good. However, most healthy women would prefer to labour and give birth physiologically if possible ${ }^{16}$ and this outcome is most likely to be associated, as outlined in the second paper of this three-part Series, with optimal wellbeing for both mother and baby in the short and longer term, as well as being more sustainable for healthcare systems. ${ }^{2}$ Qualitative and effectiveness data suggest that interventions that prioritise positive human relationships, promote respectful and collaborative multidisciplinary teamwork and address clinician beliefs and attitudes, and women's fear of labour pain and of poor quality of care, might be effective in reducing unnecessary CS and/or safely increasing physiological labour and birth. These include labour companionship, midwife-led continuity of care, midwife-led units, antenatal education, training and implementation of evidence based guidelines at point of care along with mandatory second opinion

Comparing CS rates in a standardised, meaningful, and action-oriented manner (such as through the Robson groups criteria) is critical. ${ }^{61,118}$ Where overuse is identified, the issue should be brought to the attention of the public in general, and should be high on the political agendas of countries with disproportionately high rates, especially where this occurs in parallel with underuse for some population groups. Reduction strategies require interventions that take account of the drivers identified in this paper, and that recognise the need to influence change in the beliefs and attitudes of providers, service users, and societies.

APB, MT, CK, NO and SD conceived and drafted the outline of the manuscript. APB, MT, CK, NO and SD wrote the first draft of the manuscript. APB and NO conceived and constructed table 1. CK conceived figure 1 with contributions from APB and SD. AM, MRT, JZ, MO, SZW and AMG contributed with substantial comments to the writing of the manuscript. APB, MT, CK, AM, NO, MRT, JZ, MO, SZW, AMG and SD read and approved the final manuscript. 
473 APB, CK, NO and SD had full access to the data from the systematic reviews. APB, MT and SD had 474 final responsibility for submission of the manuscript.

475

476

477

478

479 


\section{Panel 1: Search strategies \\ Cochrane systematic review}

We searched CENTRAL, MEDLINE, Embase, CINAHL and two trials registers (International Clinical Trials Registry Platform [ICTRP] and ClinicalTrials.gov) in August 2014, February 2017 and March 2018. Search strategies were comprised of keywords and controlled vocabulary terms (Medical Subject Heading [MeSH]) for $\mathrm{CS}$ and targeted non-clinical interventions. Searches for this update aimed to retrieve studies published since 2010 (i.e. the date of the searches in the previous version of the Cochrane review). The search terms were revised to increase specificity by analysing the titles, abstracts and MEDLINE index terms of the included studies from the previous version of the review using various text analysis tools (TerMine,Voyant Tools and Yale MeSH Analyzer). We applied no language limitations in the searches. We also searched reference lists of trials and related reviews, websites of relevant organisations, and contacted authors for additional articles. The complete search strategy is presented in the full review.

\section{Qualitative evidence syntheses}

Search strategies for electronic databases were developed building on preliminary scoping searches, terms used by existing quantitative reviews of interventions to reduce unnecessary CS, guidelines developed by the Cochrane Qualitative Research Methods Group, and papers detailing strategies for optimising the identification of qualitative studies in CINAHL, MEDINE, EMBASE and PsycINFO. CINAHL, MEDLINE, PsycINFO, EMBASE, Global Index Medicus, POPLINE, and African Journals Online were searched for eligible studies published between 1 January 1985 and the date of final search (22 March 2017), to identify studies since the first WHO statement on appropriate technology for childbirth. We had no language or geographic restrictions. As retrieval of qualitative research using databases alone is limited, the reference lists of all the included studies and existing quantitative reviews were back and citation chained. In addition, key articles cited by multiple authors (citation pearls) were checked on Google Scholar. The authors of published protocols were also contacted. Complete search strategies are presented in the individual reviews. 
489 Figure 1: Schematic representation of women, societal, providers and organisation factors affecting 490 CS rates at local level and enclosing the obstetric and clinical factors that also affect the rate of CS 491 births represented in the middle by the Robson's 10-group classification.

492 


\section{References}

1. WHO Statement on Caesarean Section Rates. Geneva: World Health Organization; 2015 (WHO/RHR/15.02).

2. [Paper 2 in this series] Sandall J, Tribe RM, Avery L, al. e. Short and long-term impacts/or effects of caesarean section on the health of women and children. Lancet 2018; commissioned. Lancet 2018; commissioned.

3. Gibbons L, Belizan JM, Lauer JA, Betran AP, Merialdi M, Althabe F. Inequities in the use of cesarean section deliveries in the world. Am J Obstet Gynecol 2012; 206(4): 331 e1-19.

4. Cavallaro FL, Cresswell JA, Ronsmans C. Obstetricians' Opinions of the Optimal Caesarean Rate: A Global Survey. PLoS One 2016; 11(3): e0152779.

5. Betrán AP, Ye J, Moller AB, Zhang J, Gulmezoglu AM, Torloni MR. The increasing trend in caesarean section rates: Global, regional and national estimates: 1990-2014. PLOS ONE 2016; 11(2): e0148343.

6. Habiba $M$, Kaminski $M$, Da Fre $M$, et al. Caesarean section on request: a comparison of obstetricians' attitudes in eight European countries. BJOG 2006; 113(6): 647-56.

7. Main EK, Morton CH, Melsop K, Hopkins D, Giuliani G, Gould JB. Creating a public agenda for maternity safety and quality in cesarean delivery. Obstet Gynecol 2012; 120(5): 1194-8.

8. [Paper 1 in this series] Boerma T, Ronsmans C, Barros AJ, al. e. The global epidemiology of caesarean sections. Lancet 2018; commissioned.

9. Boatin $A A$, Schlotheuber $A$, Betran $A P$, et al. Within country inequalities in caesarean section rates: observational study of 72 low and middle income countries. $B M J$ 2018; 360: k55.

10. Kabir AA, Steinmann WC, Myers L, et al. Unnecessary cesarean delivery in Louisiana: an analysis of birth certificate data. Am J Obstet Gynecol 2004; 190(1): 10-9; discussion 3A.

11. Koroukian SM, Trisel B, Rimm AA. Estimating the proportion of unnecessary Cesarean sections in Ohio using birth certificate data. J Clin Epidemiol 1998; 51(12): 1327-34.

12. Chen I, Opiyo N, Tavender E, et al. Non-clinical interventions for reducing unnecessary caesarean section. Cochrane Database of Systematic Reviews [in submission] 2018.

13. Robson SJ, Tan WS, Adeyemi A, Dear KBG. Estimating the rate of cesarean section by maternal request: Anonymous survey of obstetricians in Australia. Birth 2009; 36(3): 208-12.

14. Weaver JJ, Statham H, Richards M. Are there "unnecessary" cesarean sections? Perceptions of women and obstetricians about cesarean sections for nonclinical indications. Birth 2007; 34(1): 32-41.

15. Bettes BA, Coleman VH, Zinberg S, et al. Cesarean delivery on maternal request: obstetriciangynecologists' knowledge, perception, and practice patterns. Obstet Gynecol 2007; 109(1): 57-66.

16. Mazzoni A, Althabe F, Liu NH, et al. Women's preference for caesarean section: a systematic review and meta-analysis of observational studies. BJOG 2011; 118(4): 391-9.

17. McCourt C, Weaver J, Statham H, Beake S, Gamble J, Creedy DK. Elective cesarean section and decision making: a critical review of the literature. Birth 2007; 34(1): 65-79.

18. Mi J, Liu F. Rate of caesarean section is alarming in China. Lancet 2014; 383(9927): 1463-4.

19. Buyukbayrak EE, Kaymaz O, Kars B, et al. Caesarean delivery or vaginal birth: preference of Turkish pregnant women and influencing factors. Journal of obstetrics and gynaecology : the journal of the Institute of Obstetrics and Gynaecology 2010; 30(2): 155-8.

20. Nieminen K, Wijma K, Johansson S, et al. Severe fear of childbirth indicates high perinatal costs for Swedish women giving birth to their first child. Acta Obstet Gynecol Scand 2017; 96(4): 438-46. 
21. Yilmaz SD, Bal MD, Beji NK, Uludag S. Women's Preferences of Method of Delivery and Influencing Factors. Iranian Red Crescent medical journal 2013; 15(8): 683-9.

22. Fuglenes D, Aas E, Botten G, Oian P, Kristiansen IS. Why do some pregnant women prefer cesarean? The influence of parity, delivery experiences, and fear. Am J Obstet Gynecol 2011; 205(1): 45 e1-9.

23. Kringeland T, Daltveit AK, Moller A. What characterizes women in Norway who wish to have a caesarean section? Scandinavian journal of public health 2009; 37(4): 364-71.

24. Long $Q$, Kingdon $C$, Yand F, et al. Preference for caesarean section in China: A mixed-methods systematic review of women's and healthcare providers' views and experience. PLoS Medicine (in press) 2018.

25. Bracken JN, Dryfhout VL, Goldenhar LM, Pauls RN. Preferences and concerns for delivery: an antepartum survey. Int Urogynecol J Pelvic Floor Dysfunct 2008; 19(11): 1527-31.

26. Ghotbi F, Akbari Sene A, Azargashb E, et al. Women's knowledge and attitude towards mode of delivery and frequency of cesarean section on mother's request in six public and private hospitals in Tehran, Iran, 2012. The journal of obstetrics and gynaecology research 2014; 40(5): 1257-66.

27. Hopkins K. Are Brazilian women really choosing to deliver by cesarean? Soc Sci Med 2000; 51(5): 725-40.

28. Loke AY, Davies L, Li SF. Factors influencing the decision that women make on their mode of delivery: the Health Belief Model. BMC Health Serv Res 2015; 15: 274.

29. Torloni MR, Betran AP, Montilla P, et al. Do Italian women prefer cesarean section? Results from a survey on mode of delivery preferences. BMC Pregnancy Childbirth 2013; 13: 78.

30. Pang MW, Lee TS, Leung AK, Leung TY, Lau TK, Leung TN. A longitudinal observational study of preference for elective caesarean section among nulliparous Hong Kong Chinese women. BJOG 2007; 114(5): 623-9.

31. Hug I, Chattopadhyay C, Mitra GR, Kar Mahapatra RM, Schneider MC. Maternal expectations and birth-related experiences: a survey of pregnant women of mixed parity from Calcutta, India. Int J Obstet Anesth 2008; 17(2): 112-7.

32. Dweik D, Girasek E, Toreki A, Meszaros G, Pal A. Women's antenatal preferences for delivery route in a setting with high cesarean section rates and a medically dominated maternity system. Acta Obstet Gynecol Scand 2014; 93(4): 408-15.

33. Kasai KE, Nomura RM, Benute GR, de Lucia MC, Zugaib M. Women's opinions about mode of birth in Brazil: a qualitative study in a public teaching hospital. Midwifery 2010; 26(3): 319-26.

34. Schantz C, Sim KL, Petit V, Rany H, Goyet S. Factors associated with caesarean sections in Phnom Penh, Cambodia. Reprod Health Matters 2016; 24(48): 111-21.

35. Karlstrom A, Nystedt A, Johansson M, Hildingsson I. Behind the myth--few women prefer caesarean section in the absence of medical or obstetrical factors. Midwifery 2011; 27(5): 620-7.

36. Tschudin S, Alder J, Hendriksen S, et al. Pregnant women's perception of cesarean section on demand. J Perinat Med 2009; 37(3): 251-6.

37. Hogberg U, Lynoe N, Wulff M. Cesarean by choice? Empirical study of public attitudes. Acta Obstet Gynecol Scand 2008; 87(12): 1301-8.

38. Fenwick J, Staff L, Gamble J, Creedy DK, Bayes S. Why do women request caesarean section in a normal, healthy first pregnancy? Midwifery 2010; 26(4): 394-400.

39. Bryant J, Porter M, Tracy SK, Sullivan EA. Caesarean birth: consumption, safety, order, and good mothering. Soc Sci Med 2007; 65(6): 1192-201.

40. Fioretti B, Reiter M, Betran A, Torloni M. Googling caesarean section: a survey on the quality of the information available on the Internet. BJOG 2014.

41. Torloni M, Campos Mansilla B, Merialdi M, Betran A. What do popular Spanish women's magazines say about caesarean section? A 21-year survey. BJOG 2014. 
42. Bantan N, Abenhaim HA. Vaginal births after caesarean: what does Google think about it? Women Birth 2015; 28(1): 21-4.

43. Amyx M, Gibbons $L$, Xiong $X$, et al. Sources of influence on pregnant women's preferred mode of delivery in Buenos Aires, Argentina. Birth 2018; 45(1): 71-8.

44. Johansson M, Radestad I, Rubertsson C, Karlstrom A, Hildingsson I. Few fathers-to-be prefer caesarean section for the birth of their baby. BJOG 2010; 117(6): 761-4.

45. Kingdon C, Downe S, Betrán AP. What do women and communities say about educational interventions targeted at them to reduce unnecessary caesarean section? A qualitative evidence synthesis. Reproductive Health (in submission) 2018.

46. Ji H, Jiang $H$, Yang L, Qian X, Tang S. Factors contributing to the rapid rise of caesarean section: a prospective study of primiparous Chinese women in Shanghai. BMJ Open 2015; 5(11): e008994.

47. Studdert DM, Mello MM, Gawande AA, et al. Claims, errors, and compensation payments in medical malpractice litigation. N Engl J Med 2006; 354(19): 2024-33.

48. Kravitz RL, Rolph JE, McGuigan K. Malpractice claims data as a quality improvement tool. I. Epidemiology of error in four specialties. JAMA 1991; 266(15): 2087-92.

49. Jena AB, Schoemaker L, Bhattacharya J, Seabury SA. Physician spending and subsequent risk of malpractice claims: observational study. BMJ 2015; 351: h5516.

50. Asher E, Dvir S, Seidman DS, et al. Defensive medicine among obstetricians and gynecologists in tertiary hospitals. PLoS One 2013; 8(3): e57108.

51. Grytten J, Skau I, Sorensen R. The impact of the mass media on obstetricians' behavior in Norway. Health Policy 2017; 121(9): 986-93.

52. Bettes BA, Strunk AL, Coleman VH, Schulkin J. Professional liability and other career pressures: impact on obstetrician-gynecologists' career satisfaction. Obstet Gynecol 2004; 103(5 Pt 1): 967-73.

53. Litorp H, Mgaya A, Mbekenga CK, Kidanto HL, Johnsdotter S, Essen B. Fear, blame and transparency: Obstetric caregivers' rationales for high caesarean section rates in a lowresource setting. Soc Sci Med 2015; 143: 232-40.

54. Localio AR, Lawthers AG, Bengtson JM, et al. Relationship between malpractice claims and cesarean delivery. JAMA 1993; 269(3): 366-73.

55. Dweik D, Girasek E, Meszaros G, Toreki A, Kereszturi A, Pal A. Non-medical determinants of cesarean section in a medically dominated maternity system. Acta Obstet Gynecol Scand 2014; 93(10): 1025-33.

56. Gomes UA, Silva AA, Bettiol H, Barbieri MA. Risk factors for the increasing caesarean section rate in Southeast Brazil: a comparison of two birth cohorts, 1978-1979 and 1994. Int J Epidemiol 1999; 28(4): 687-94.

57. Murray SF. Relation between private health insurance and high rates of caesarean section in Chile: qualitative and quantitative study. BMJ 2000; 321(7275): 1501-5.

58. Al Rifai RH. Trend of caesarean deliveries in Egypt and its associated factors: evidence from national surveys, 2005-2014. BMC Pregnancy Childbirth 2017; 17(1): 417.

59. Brazil Ministry of Health. SUS Hospital procedures. [Procedimentos hospitalares do SUS]. 2015 (http://tabnet.datasus.gov.br/cgi/deftohtm.exe?sih/cnv/qiuf.def, accessed 12 Jul 2016).

60. Brazil Ministry of Health. MS/SVS/DASIS: National Data System on Live Births - SINASC [Sistema de Informações de Nascidos Vivos - SINASC]. 2016. http://tabnet.datasus.gov.br/cgi/deftohtm.exe?sinasc/cnv/nvuf.defAccessed 12 Jul 2016.).

61. Betrán AP, Torloni MR, Zhang J, Gülmezoglu AM, Section aftWWGoC. Commentary: WHO Statement on caesarean section rates. BJOG 2016; 123(5): 667-70.

62. Robson M, Hartigan L, Murphy M. Methods of achieving and maintaining an appropriate caesarean section rate. Best Pract Res Clin Obstet Gynaecol 2013; 27: 297-308.

63. WHO recommendations on antenatal care for a positive pregnancy experience. Geneva, Switzerland: World Health Organization; 2016. 
64. WHO recommendations: intrapartum care for a positive childbirth experience. Geneva, Switzerland: World Health Organization; 2018.

65. WHO recommendations on non-clinical interventions to reduce unnecessary caesareans births. Geneva, Switzerland, 2018 (in press).

66. Walker KF, Bugg GJ, Macpherson M, et al. Randomized Trial of Labor Induction in Women 35 Years of Age or Older. N Engl J Med 2016; 374(9): 813-22.

67. Saccone G, Berghella V. Induction of labor at full term in uncomplicated singleton gestations: a systematic review and metaanalysis of randomized controlled trials. Am J Obstet Gynecol 2015; 213(5): 629-36.

68. Mishanina E, Rogozinska E, Thatthi T, Uddin-Khan R, Khan KS, Meads C. Use of labour induction and risk of cesarean delivery: a systematic review and meta-analysis. CMAJ 2014; 186(9): 665-73.

69. National Institutes of Health. Induced labor after 39 weeks in healthy women may reduce need for $C$ section (published online Feb 1). 2018.

70. Middleton $P$, Shepherd E, Crowther CA. Induction of labour for improving birth outcomes for women at or beyond term. Cochrane Database Syst Rev 2018; 5: CD004945.

71. Grobman $W$ for the Aunice Kennedy Shriver National Institute of Child Health and Human Development Maternal-Fetal Medicine Units Network. A randomized trial of elective induction of labour at 39 weeks compared with expectant management of low-risk nulliparous women. Am J Obstet Gynecol 2018; Abstract LB01: S601.

72. Main E. Comments on the Arrive Trial. https://www.cmqcc.org/sites/default/files/Arrive\%20Trial\%20Statement\%20Final.pdf: California Maternal QUality Care Collaborative, 2018.

73. Brown HC, Paranjothy S, Dowswell T, Thomas J. Package of care for active management in labour for reducing caesarean section rates in low-risk women. Cochrane Database Syst Rev 2013; (9): CD004907.

74. Bohren MA, Hofmeyr GJ, Sakala C, Fukuzawa RK, Cuthbert A. Continuous support for women during childbirth. Cochrane Database Syst Rev 2017; 7: CD003766.

75. Hutton EK, Hofmeyr GJ, Dowswell T. External cephalic version for breech presentation before term. Cochrane Database Syst Rev 2015; (7): CD000084.

76. Hofmeyr GJ, Hannah M, Lawrie TA. Planned caesarean section for term breech delivery. Cochrane Database Syst Rev 2015; (7): CD000166.

77. Hannah ME, Hannah WJ, Hewson SA, Hodnett ED, Saigal S, Willan AR. Planned caesarean section versus planned vaginal birth for breech presentation at term: a randomised multicentre trial. Term Breech Trial Collaborative Group. Lancet 2000; 356(9239): 1375-83.

78. Whyte $\mathrm{H}$, Hannah ME, Saigal $\mathrm{S}$, et al. Outcomes of children at 2 years after planned cesarean birth versus planned vaginal birth for breech presentation at term: the International Randomized Term Breech Trial. Am J Obstet Gynecol 2004; 191(3): 864-71.

79. Bjellmo S, Andersen GL, Martinussen MP, et al. Is vaginal breech delivery associated with higher risk for perinatal death and cerebral palsy compared with vaginal cephalic birth? Registry-based cohort study in Norway. BMJ Open 2017; 7(4): e014979.

80. Dodd JM, Crowther CA, Huertas E, Guise JM, Horey D. Planned elective repeat caesarean section versus planned vaginal birth for women with a previous caesarean birth. Cochrane Database Syst Rev 2013; (12): CD004224.

81. Clarke M, Savage $G$, Smith V, et al. Improving the organisation of maternal health service delivery and optimising childbirth by increasing vaginal birth after caesarean section through enhanced women-centred care (OptiBIRTH trial): study protocol for a randomised controlled trial (ISRCTN10612254). Trials 2015; 16: 542.

82. Lomas J, Enkin M, Anderson GM, Hannah WJ, Vayda E, Singer J. Opinion leaders vs audit and feedback to implement practice guidelines. Delivery after previous cesarean section. JAMA 1991; 265(17): 2202-7. 
83. Committee on Practice Bulletins-Obstetrics. Practice Bulletin No. 184: Vaginal Birth After Cesarean Delivery. Obstet Gynecol 2017; 130: e217-33.

84. Royal College of Obstetricians \& Gynecologist. Birth after Previous Caesarean Birth (Green-top Guideline No. 45). 2017.

85. Sandall J, Soltani H, Gates S, Shennan A, Devane D. Midwife-led continuity models versus other models of care for childbearing women. Cochrane Database Syst Rev 2016; (4): CD004667.

86. Alfirevic Z, Devane D, Gyte GM, Cuthbert A. Continuous cardiotocography (CTG) as a form of electronic fetal monitoring (EFM) for fetal assessment during labour. Cochrane Database Syst Rev 2017; 2: CD006066.

87. Kingdon C, Downe S, Betrán AP. Non-clinical interventions to reduce unnecessary caesarean section targeted at organisations, facilities and systems: A qualitative evidence synthesis of stakeholders' views PLOS ONE (in submission) 2018.

88. Kingdon C, Downe S, Betrán AP. Too much caesarean section? Health professionals' views of interventions to reduce unnecessary caesarean section targeted at them: A qualitative evidence synthesis. PLOS ONE (in submission) 2018.

89. Bastani F, Hidarnia A, Montgomery KS, Aguilar-Vafaei ME, Kazemnejad A. Does relaxation education in anxious primigravid Iranian women influence adverse pregnancy outcomes?: a randomized controlled trial. The Journal of perinatal \& neonatal nursing 2006; 20(2): 138-46.

90. Feinberg ME, Roettger ME, Jones DE, Paul IM, Kan ML. Effects of a psychosocial couple-based prevention program on adverse birth outcomes. Maternal and child health journal 2015; 19(1): 102-11.

91. Valiani M, Haghighatdana Z, Ehsanpour S. Comparison of childbirth training workshop effects on knowledge, attitude, and delivery method between mothers and couples groups referring to Isfahan health centers in Iran. Iranian journal of nursing and midwifery research 2014; 19(6): 653-8.

92. Rouhe H, Salmela-Aro K, Toivanen R, Tokola M, Halmesmaki E, Saisto T. Obstetric outcome after intervention for severe fear of childbirth in nulliparous women - randomised trial. BJOG 2013; 120(1): 75-84.

93. Navaee M, Abedian Z. Effect of role play education on primiparous women's fear of natural delivery and their decision on the mode of delivery. Iranian journal of nursing and midwifery research 2015; 20(1): 40-6.

94. Eden KB, Perrin NA, Vesco KK, Guise JM. A randomized comparative trial of two decision tools for pregnant women with prior cesareans. Journal of obstetric, gynecologic, and neonatal nursing : JOGNN 2014; 43(5): 568-79.

95. Fraser W, Maunsell E, Hodnett E, Moutquin JM. Randomized controlled trial of a prenatal vaginal birth after cesarean section education and support program. Am J Obstet Gynecol 1997; 176(2): 419-25.

96. Shorten A, Fagerlin A, Illuzzi J, et al. Developing an Internet-Based Decision Aid for Women Choosing Between Vaginal Birth After Cesarean and Planned Repeat Cesarean. Journal of midwifery \& women's health 2015; 60(4): 390-400.

97. Althabe F, Belizan JM, Villar J, et al. Mandatory second opinion to reduce rates of unnecessary caesarean sections in Latin America: a cluster randomised controlled trial. Lancet 2004; 363(9425): 1934-40.

98. Chaillet $\mathrm{N}$, Dumont $\mathrm{A}$, Abrahamowicz $\mathrm{M}$, et al. A cluster-randomized trial to reduce cesarean delivery rates in Quebec. N Engl J Med 2015; 372(18): 1710-21.

99. Rosenstein MG, Nijagal M, Nakagawa S, Gregorich SE, Kuppermann M. The Association of Expanded Access to a Collaborative Midwifery and Laborist Model With Cesarean Delivery Rates. Obstet Gynecol 2015; 126(4): 716-23.

100. Keeler EB, Fok T. Equalizing physician fees had little effect on cesarean rates. Medical care research and review : MCRR 1996; 53(4): 465-71. 
101. Lo JC. Financial incentives do not always work: an example of cesarean sections in Taiwan. Health Policy 2008; 88(1): 121-9.

102. Hulscher ME, Schouten LM, Grol RP, Buchan H. Determinants of success of quality improvement collaboratives: what does the literature show? BMJ quality \& safety 2013; 22(1): 19-31.

103. Mazzucca S, Tabak RG, Pilar M, et al. Variation in Research Designs Used to Test the Effectiveness of Dissemination and Implementation Strategies: A Review. Front Public Health 2018; $6: 32$.

104. Portela MC, Pronovost PJ, Woodcock T, Carter P, Dixon-Woods M. How to study improvement interventions: a brief overview of possible study types. BMJ quality \& safety 2015; 24(5): 32536.

105. Brown CH, Curran G, Palinkas LA, et al. An Overview of Research and Evaluation Designs for Dissemination and Implementation. Annu Rev Public Health 2017; 38: 1-22.

106. Brown $\mathrm{CH}$, Ten Have TR, Jo B, et al. Adaptive designs for randomized trials in public health. Annu Rev Public Health 2009; 30: 1-25.

107. Liu X, Lynch CD, Cheng WW, Landon MB. Lowering the high rate of caesarean delivery in China: an experience from Shanghai. BJOG 2016; 123(10): 1620-8.

108. Yu Y, Zhang X, Sun C, Zhou H, Zhang Q, Chen C. Reducing the rate of cesarean delivery on maternal request through institutional and policy interventions in Wenzhou, China. PLoS One 2017; 12(11): e0186304.

109. Ayres-De-Campos D, Cruz J, Medeiros-Borges C, Costa-Santos C, Vicente L. Lowered national cesarean section rates after a concerted action. Acta Obstet Gynecol Scand 2015; 94(4): 3918.

110. Borem P, Ferreira JB, da Silva UJ, Valerio Junior J, Orlanda CM. [Increasing the percentage of vaginal birth in the private sector in Brazil through the redesign of care model]. Revista brasileira de ginecologia e obstetricia : revista da Federacao Brasileira das Sociedades de Ginecologia e Obstetricia 2015; 37(10): 446-54.

111. Smith H, Peterson N, Lagrew D, Main EK. Toolkit to Support Vaginal Birth and Reduce Primary Cesareans: A Quality Improvement Toolkit. Stanford, CA: California Maternal Quality Care Collaborative. . 2016.

112. Long $Q$, Allanson ER, Pontre J, Tuncalp O, Hofmeyr GJ, Gulmezoglu AM. Onsite midwife-led birth units (OMBUs) for care around the time of childbirth: a systematic review. BMJ Glob Health 2016; 1(2): e000096.

113. Oleske DM, Glandon GL, Tancredi DJ, Nassirpour M, Noak JR. Information dissemination and the cesarean birth rate. The Illinois experience. Int J Technol Assess Health Care 1992; 8(4): 708-18.

114. Kim CY, Ko SK, Kim KY. Are league tables controlling epidemic of caesarean sections in South Korea? BJOG 2005; 112(5): 607-11.

115. Khang YH, Yun SC, Jo MW, Lee MS, Lee SI. Public release of institutional Cesarean section rates in South Korea: which women were aware of the information? Health Policy 2008; 86(1): 10 16.

116. Shepperd S, Lewin S, Straus $S$, et al. Can we systematically review studies that evaluate complex interventions? PLoS medicine 2009; 6(8): e1000086.

117. Auerbach AD, Landefeld CS, Shojania KG. The tension between needing to improve care and knowing how to do it. N Engl J Med 2007; 357(6): 608-13.

118. Torloni MR, Betran AP, Souza JP, et al. Classifications for cesarean section: a systematic review. PLOS ONE 2011; 6(1): e14566. 